\title{
FOXO3 programs tumor-associated DCs to become tolerogenic in human and murine prostate cancer
}

\author{
Stephanie K. Watkins, ${ }^{1}$ Ziqiang Zhu, ${ }^{1}$ Elena Riboldi, ${ }^{2}$ Kim A. Shafer-Weaver, ${ }^{1,3}$ \\ Katherine E.R. Stagliano, ${ }^{1}$ Martha M. Sklavos, ${ }^{1}$ Stefan Ambs, ${ }^{4}$ Hideo Yagita, ${ }^{5}$ and Arthur A. Hurwitz ${ }^{1}$ \\ ${ }^{1}$ Tumor Immunity and Tolerance Section, Laboratory of Molecular Immunoregulation and 2Laboratory of Experimental Immunology, \\ Cancer and Inflammation Program, National Cancer Institute-Frederick (NCI-Frederick), Frederick, Maryland, USA. ${ }^{3}$ SAIC-Frederick Inc., \\ Frederick, Maryland, USA. ${ }^{4}$ Breast and Prostate Cancer Unit, Laboratory of Human Carcinogenesis, NCI, Frederick, Maryland, USA. \\ ${ }^{5}$ Department of Immunology, Juntendo University School of Medicine, Tokyo, Japan.
}

\begin{abstract}
The limited success of cancer immunotherapy is often attributed to the loss of antigen-specific $T$ cell function in situ. However, the mechanism for this loss of function is unknown. In this study, we describe a population of tumor-associated DCs (TADCs) in both human and mouse prostate cancer that tolerizes and induces suppressive activity in tumor-specific $T$ cells. In tumors from human prostate cancer patients and transgenic adenocarcinoma of the mouse prostate (TRAMP) mice, TADCs expressed elevated levels of FOXO3 and Foxo3, respectively, which correlated with expression of suppressive genes that negatively regulate $T$ cell function. Silencing FOXO3 and Foxo3 with siRNAs abrogated the ability of human and mouse TADCs, respectively, to tolerize and induce suppressive activity by $T$ cells. Silencing Foxo3 in mouse TADCs was also associated with diminished expression of tolerogenic mediators, such as indoleamine-2,3-dioxygenase, arginase, and TGF- $\beta$, and upregulated expression of costimulatory molecules and proinflammatory cytokines. Importantly, transfer of tumor-specific $\mathrm{CD}^{+}$Th cells into TRAMP mice abrogated TADC tolerogenicity, which was associated with reduced Foxo3 expression. These findings demonstrate that FOXO3 may play a critical role in mediating TADC-induced immune suppression. Moreover, our results identify what we believe to be a novel target for preventing CTL tolerance and enhancing immune responses to cancer by modulating the immunosuppressive activity of TADCs found in the tumor microenvironment.
\end{abstract}

\section{Introduction}

Many populations of suppressive cells have been attributed to tumor growth, including macrophages and other myeloid-derived suppressor cells, regulatory T cells, and, more recently, DCs (1-3). Subpopulations of tumor-associated DCs (TADCs) that have been described include conventional DCs (cDCs) and plasmacytoid DCs (pDCs) as well as other indoleamine 2,3-dioxygenase ${ }^{+} /$ $\mathrm{CD} \alpha^{+}\left(\mathrm{IDO}^{+} / \mathrm{CD} \alpha^{+}\right)$DCs $(4-7)$. Immune suppression induced by DCs has been attributed to catabolic enzymes such as IDO, which targets tryptophan, and arginase, which eventually leads to a downregulation of $\mathrm{CD} 3 \xi$; in each case, the result is the inhibition of T cell activation $(5,8-10)$. DCs can also express cell surface ligands, such as programmed cell death 1 ligand 1 (PD-L1) and PD-L2, or cytokines (e.g., IL-10 and TGF- $\beta)(11,12)$ that can suppress $\mathrm{T}$ cell responses.

DCs are known to be associated with the induction of $\mathrm{T}$ cell tolerance in cancer. Tissue-specific or tumor antigens may be taken up by resting DCs and cross-presented, resulting in the tolerization of $\mathrm{T}$ cells (13-15). Furthermore, others have reported that pDCs residing in tumor may deliver poor or tolerogenic signals to $\mathrm{T}$ cells (16-20). However, we and others have demonstrated that TADCs can be "licensed" in situ to support antitumor immunity $(21,22)$. Therefore a better understanding of the mechanisms that regulate DC function in tumors will aid in the development of more effective cancer vaccines.

Conflict of interest: The authors have declared that no conflict of interest exists. Citation for this article: J Clin Invest. 2011;121(4):1361-1372. doi:10.1172/JCI44325.
The molecular mechanisms that control DC dysfunction are complex and are a function of the tumor microenvironment. While many signaling pathways are dysregulated in tumor-infiltrating leukocytes, the signals that induce DC dysfunction require further investigation. The JAK/STAT families of molecules are critical components in cell survival, proliferation, and differentiation; several studies have identified activation of STAT3 as one component of immune suppression in cancer $(23,24)$. FOXO3 is another transcriptional regulator that was originally identified as a tumor suppressor but was recently associated with DC function $(25,26)$. In that study, it was suggested that FOXO3 controls DC stimulatory capacity. However, a role for FOXO3 in controlling DC function in cancer and, in particular, the tolerogenic function of DCs in cancer has not been identified.

In the current report, we describe for what we believe to be the first time similar characteristics and functional capabilities of DCs isolated from prostate tumor tissue in mice and humans. Human TADCs had a phenotype consistent with pDCs and tolerized T cells. Similarly, TADCs from transgenic adenocarcinoma of the mouse prostate (TRAMP) mice were highly tolerogenic and induced suppressive activity in tumor-specific T cells. Furthermore, our studies identified $\mathrm{FOXO} 3$ as a critical signaling molecule in the tolerogenic programming of human and TRAMP TADCs. Silencing Foxo3 expression using siRNAs ablated the immunosuppressive functions of both human and murine TADCs. Given this regulation that we believe to be novel of TADC tolerogenicity by FOXO3, we propose that this transcriptional regulator can serve as a new target for enhancing cancer immunotherapy. 
A

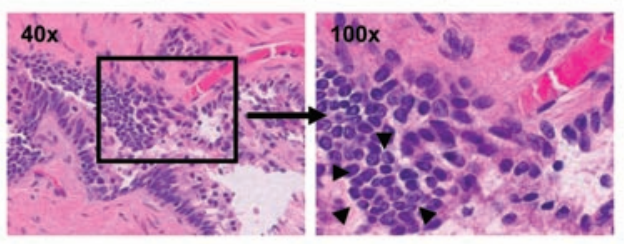

C
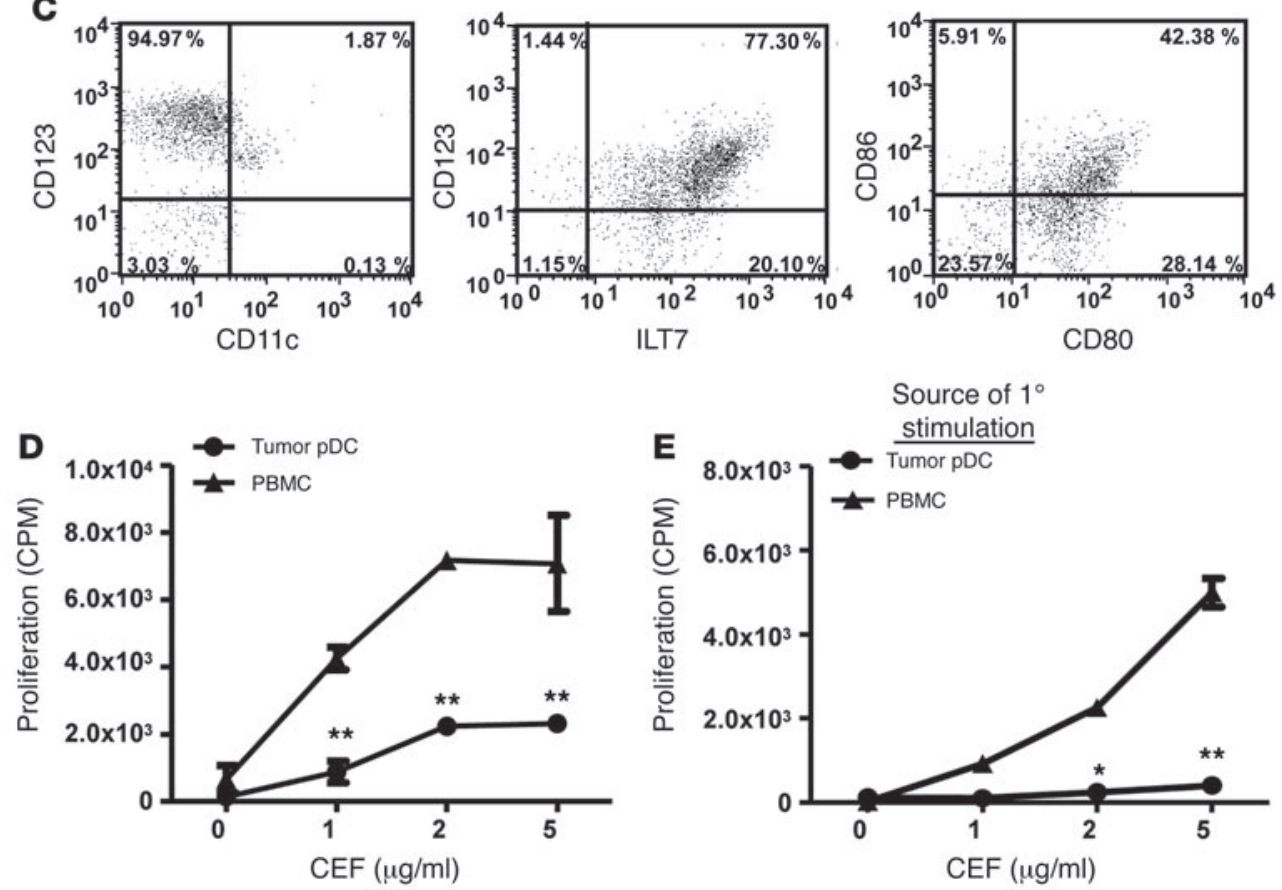

Source of $1^{\circ}$

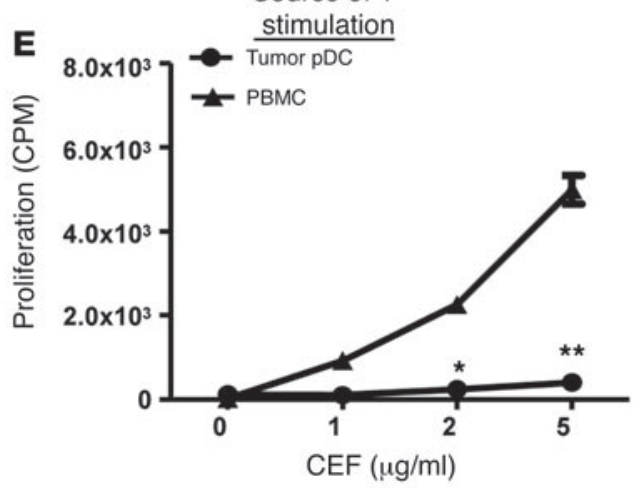

Figure 1

TADCs infiltrate human prostate tumors and tolerize T cells. (A) Prostate tumors are infiltrated by inflammatory cells (arrowheads). (B) Enriched TADCs were stained with a modified Wright-Giemsa stain after cytospin and $(\mathbf{C})$ were analyzed for $\mathrm{pDC}$ surface markers and costimulatory molecule expression. Numbers represent the percentages of cells present in the given quadrant. (D) Purified TADCs were used to stimulate PBMCs in vitro. (E) Peripheral blood T cells were cocultured in vitro with CEF peptide and TADCs prior to secondary stimulation with CEF and autologous PBMCs. $1^{\circ}$, primary. Data are representative of (D) 4 patient samples or $(\mathbf{E}) 2$ patient samples (mean \pm SD). Original magnification, $\times 40$ (A, left); $\times 100$ (A, right, and $\mathbf{B}) .{ }^{*} P<0.01,{ }^{* \star} P<0.001$ (Student's $t$ test). See also Supplemental Figure 1.

\section{Results}

Tolerogenic $p D C$ infiltrate buman prostate tumors. While TADCs have been previously identified in human prostate cancer specimens $(27,28)$, we sought to identify their function. Histological analyses detected strong leukocytic infiltration in biopsies of advanced prostate tumors (Figure 1A). Flow cytometric analysis of disaggregated tumor biopsies revealed that among the $\mathrm{CD} 45^{+}$cells, $63 \%$ were $\mathrm{CD}_{14}{ }^{+} / \mathrm{CD} 16^{+}$macrophages, $21 \%$ were $\mathrm{CD} 11 \mathrm{c}^{+} \mathrm{cDCs}$,

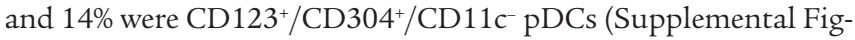
ure $1 \mathrm{~A}$; supplemental material available online with this article; doi:10.1172/JCI44325DS1). Based on their observed regulatory function (Supplemental Figure 1B), the pDC population enriched by magnetic beads coupled to anti-PTK7 or anti-CD304 (29) was characterized further. The purified cells had a plasma cell-like morphology (Figure 1B) and were $\mathrm{CD}_{123^{+}}, \mathrm{ILT}^{+}$, and $\mathrm{CD} 11 \mathrm{c}^{-}$, consistent with human $\mathrm{pDCs}$, and also expressed low levels of CD80 and CD86 (Figure 1C). To determine the ability of human prostate TADCs to stimulate $T$ cells, we cultured enriched pDCs with
CD80

autologous peripheral blood $\mathrm{T}$ cells and a pool of common viral (CMV, EBV, and flu [CEF]) antigens and measured $\mathrm{T}$ cell proliferation. Using this assay, we observed that the CD $123^{+}$pDCs from tumor biopsies (TADCs) had a lower stimulatory activity than that of autologous PBMCs (Figure 1D) or pDCs from nontumor tissue (Supplemental Figure $1 \mathrm{~B})$. Given this diminished stimulatory activity, we next assessed the tolerogenicity of TADCs by testing their ability to tolerize peripheral blood $\mathrm{T}$ cells. We designed a tolerance assay wherein 3 days after coculture with TADCs and CEF antigen, $T$ cells were harvested and restimulated with autologous PBMCs and CEF antigen, and proliferation was assessed. Unlike the strong proliferative response observed by $\mathrm{T}$ cells initially cultured with PBMCs as a source of APCs, T cells initially cultured with TADCs were unable to respond to secondary stimulation by autologous PBMCs and antigen (Figure 1E). These data demonstrate that human TADCs were tolerogenic.

TRAMP TADCs display an immunosuppressive expression profile. To study the role of TADCs in prostate cancer and the mechanisms by which they tolerize T cells, we used the experimental TRAMP model. TRAMP mice develop autochthonous prostatic tumors, due to prostate-specific expression of the SV40 T antigen (TAg). We previously reported that upon entry into the TRAMP prostate, tumor-specific T cells become tolerized and acquire suppressive function $(30,31)$. Therefore, we sought to determine whether prostate TADCs in TRAMP mice were capable of tolerizing TcR-I cells. Initially, we determined the phenotype and gene expression profile of DCs in TRAMP mice. The peripheral lymphoid tissues contained a small but discreet population of $\mathrm{B} 220^{+} \mathrm{CD} 317^{+} \mathrm{DCs}$, consistent with a $\mathrm{pDC}$ phenotype (Supplemental Figure 2, A-C). The TRAMP tumors contained a heterogeneous population of myeloid cells, the majority of which were $\mathrm{CD} 11 \mathrm{~b}^{+} / \mathrm{F} 4 / 80^{+}$tumor-associated macrophages (TAMs) (Supplemental Figure 2D). However, the predominant population of DCs were CD11c $\mathrm{C}^{+} / \mathrm{B} 220^{+} / \mathrm{BST} 2(\mathrm{CD} 317)^{+} / \mathrm{CD}_{11 b^{-}}$(Figure $2 \mathrm{~A}$ and Supplemental Figure 2D), which represented approximately $30 \%$ of the $\mathrm{CD}_{4} 5^{+}$cells in the TRAMP prostate. Interestingly, DCs with this $\mathrm{PDC}$ surface phenotype were also detected in the WT prostate tissue (Figure 2B). Perfusing the prostate tumor prior to assessing phenotype or isolating DCs did not change the total number of 

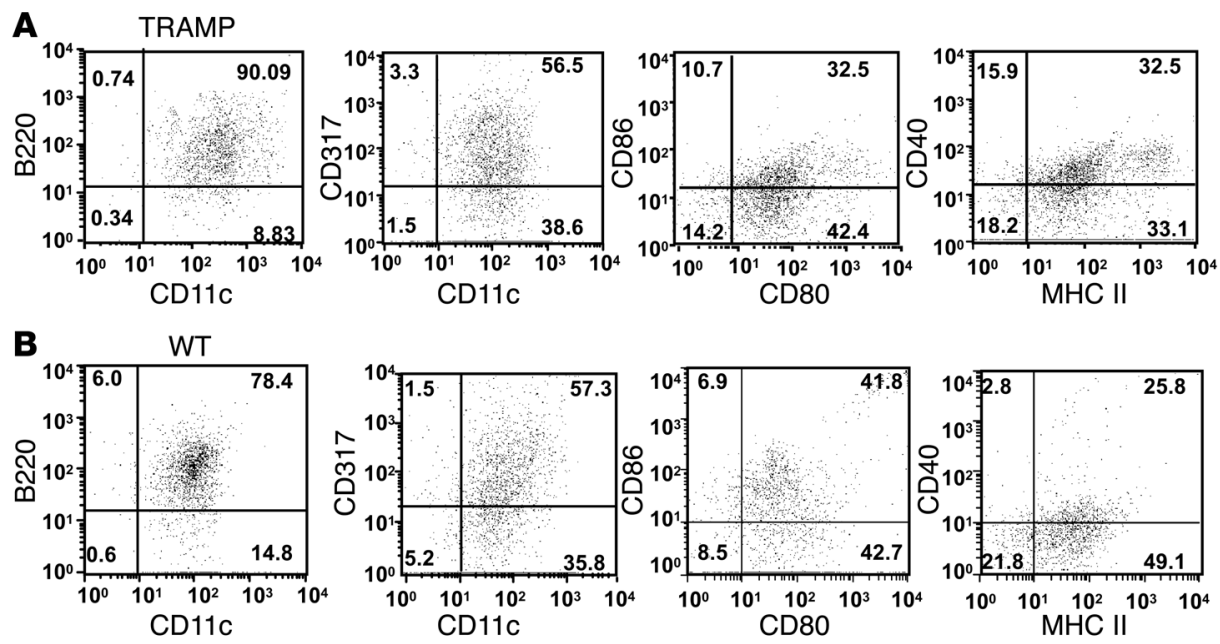

\section{Figure 2}

TRAMP TADCs and WT prostate DCs have a similar phenotype. Prostatic DCs purified from (A) TRAMP and (B) WT mice were analyzed for surface antigen expression by flow cytometry. Numbers represent the percentages of cells present in the given quadrant. Data are representative of 4 independent trials.

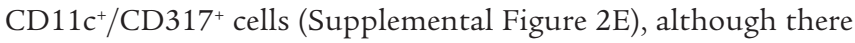
was a small decrease in $\mathrm{CD} 11 \mathrm{c}^{+} / \mathrm{F} 4 / 80^{+}$cells, presumably TAMs. Additional phenotyping revealed that WT and TRAMP prostate DCs expressed low to intermediate levels of the costimulatory molecules CD80, CD86, and CD40 as well as MHC class II, all of which are crucial for effective priming of naive T cells (Figure 2, A and B).

To obtain a more definitive understanding of gene expression by prostatic DCs, microarray analysis was used, comparing the profiles of WT and TRAMP prostate DCs. Our data demonstrated a significant upregulation of chemokine genes important for $\mathrm{T}$ cell chemotaxis (Cxcl10, Cxcl9, Ccl5), indicating that TADCs may actively recruit immune cells into the tumor (ref. 30 and Table 1). Paradoxically, TRAMP TADCs also overexpressed genes associated with T cell tolerance, including arginase (Arg1) and IDO (Ido1) (Table 1), as well as several cytokines associated with prostate cancer development that can directly suppress immune cell function $(T g f b 1)$ or promote signaling pathways associated with the growth and metastasis of prostate cancer, such as Il6 and Vegfa (Table 1). Furthermore, the microarray data also revealed upregulation of genes associated with signaling pathways, such as Jak2, Stat3, and Foxo3, in TADCs (Table 1). Quantitative real-time PCR (qrt-PCR), flow cytometric analysis, and ELISAs confirmed our microarraybased observations that genes associated with immune suppression (Ido1, Ido2, Arg1, Cd274, and Tgfb) were upregulated in TADCs (Supplemental Figure 3, A-C).

TADCs induce T cell tolerance and suppressor cell activity. Given this apparent immunosuppressive phenotype, the stimulatory capacity of DCs purified from TRAMP and WT prostates were compared using naive SV40 TAg-specific CD8 ${ }^{+}$(TcR-I) T cells as responder cells. In contrast to WT prostate DCs, which stimulated TcR-I cell proliferation, TRAMP TADCs were unable to induce a strong proliferative response by TcR-I cells (Figure 3A), suggesting that TADCs were incapable of eliciting a $\mathrm{T}$ cell immune response and, instead, may be tolerogenic.

To determine whether TRAMP TADCs tolerize TcR-I T cells, similar to the human tolerance assay, prostatic DCs and naive TcR-I $\mathrm{T}$ cells were cocultured for 4 days, after which the $\mathrm{T}$ cells were reisolated and stimulated with TAg peptide-pulsed splenic APCs. TcR-I T cells initially cultured with TRAMP TADCs did not proliferate (Figure 3B) or produce IFN- $\gamma$ (Supplemental Figure 4A) in response to secondary antigenic stimulation, whereas marked pro- liferative and cytokine responses were observed when WT prostate DCs were used as APCs for the primary stimulation (Figure 3B). Furthermore, effector TcR-I T cells that were primed in vivo (31) were also tolerized by TRAMP TADCs (Supplemental Figure 4B). Tolerance induction was antigen specific, as TRAMP TADCs tolerized TcR-I T cells without the addition of TAg, presumably due to antigen carry over from the TRAMP tumor, but were unable to tolerize melanoma antigen-specific transgenic (TcR-Mel) T cells (Supplemental Figure 4, C and D). However, TRAMP TADCs tolerized the TcR-Mel T cells when pulsed with the cognate melanoma antigen (Supplemental Figure 4D). Taken together, these data demonstrate that DCs from the TRAMP tumor were not only ineffective at priming naive $T$ cells but also tolerized naive and effector $T$ cells in an antigen-specific manner.

We previously reported that upon tumor infiltration, TcR-I cells not only become tolerized but also acquire suppressive function (31). Therefore, we next sought to determine whether TADCs induced TcR-I cells to become suppressive. As demonstrated in Figure 3C, TcR-I T cells cultured with DCs from TRAMP tumors became highly suppressive and prevented naive $\mathrm{T}$ cell proliferation (Figure 3C). In contrast, DCs purified from WT prostates did not

\section{Table 1}

Gene expression patterns in TRAMP TADCs differ from those in WT prostate DCs

\begin{tabular}{lccc}
$\begin{array}{l}\text { Chemokines/ } \\
\text { cytokines }\end{array}$ & $\begin{array}{c}\text { Fold change } \\
\text { TRAMP/WT }\end{array}$ & $\begin{array}{c}\text { Other genes } \\
\text { of interest }\end{array}$ & $\begin{array}{c}\text { Fold change } \\
\text { TRAMP/WT }\end{array}$ \\
Cxc/10 & 24.1 & Fasl & 2.4 \\
Cxcl9 & 6.2 & Ido1 & 4.5 \\
Ccl5 & 6.7 & Arg1 & 6.2 \\
II6 & 5.9 & Nos2 & 3.1 \\
Tgfb1 & 2.4 & Cd274 & 3.5 \\
Vegfa & 2.4 & Stat3 & 5.6 \\
II1b & 2.9 & Foxo3 & 4.2 \\
\hline
\end{tabular}

After purification, RNA was isolated and hybridized to an Affymetrix ST 1.0 Mouse gene array. Fold change values have corresponding $P$ values of less than 0.00001 (ANOVA). Data are representative of 4 independent microarrays for WT and TRAMP samples. $\mathrm{Cxc} / 10$ is also known as IP-10; Cxc/9 is also known as Mig; and Cc/5 is also known as RANTES. See also Supplemental Figures 2 and 3. 
A

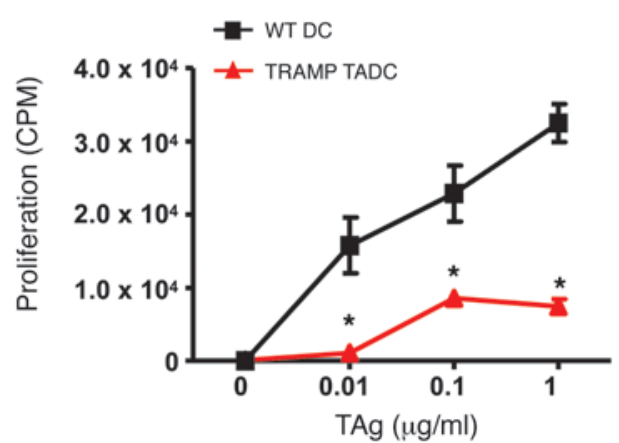

B

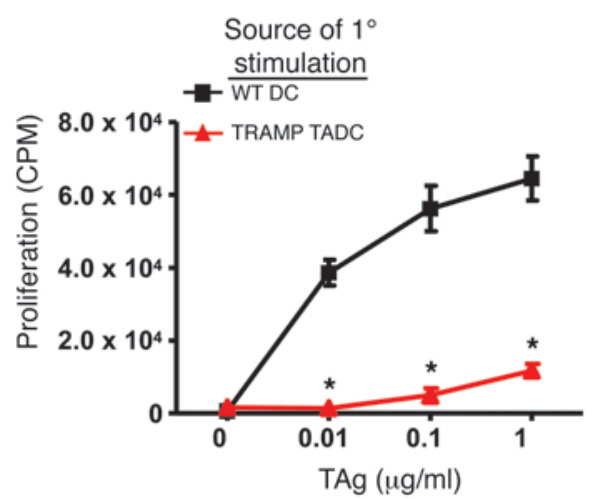

C Source of $1^{\circ}$

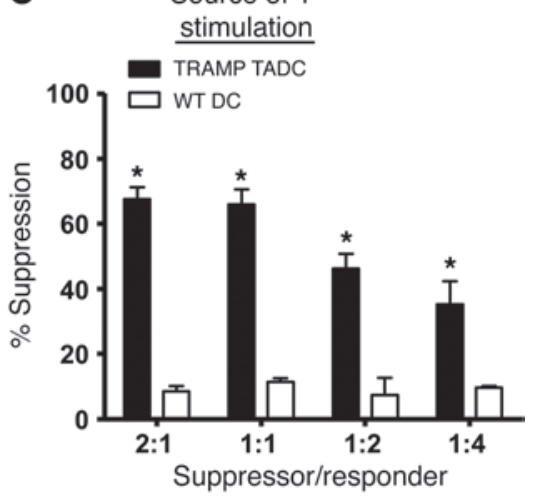

Figure 3

TRAMP TADCs are poor stimulators of $\mathrm{CD}^{+} \mathrm{T}$ cell proliferation and induce $\mathrm{T}$ cell tolerance and suppressive activity in vitro. DCs were isolated from prostate tissues using antibody-coupled magnetic beads, as described in Methods. (A) Purified DCs were used to stimulate naive CD8+ T cell proliferation in vitro. (B) TcR-I T cells were cocultured in vitro with TAg peptide and prostatic DCs from TRAMP or WT mice for 4 days prior to secondary stimulation with TAg peptide and splenic APCs. (C) TcR-I T cells cultured with TRAMP DCs were isolated after 4 days and used in a suppressor assay. ${ }^{*} P<0.0001$ WT vs. TRAMP (Student's $t$ test). Data are representative of 4 independent trials (3 WT and 3 TRAMP mice in each experiment; mean \pm SD). See also Supplemental Figure 4.

induce suppressive activity. These findings demonstrate that, like TADCs isolated from human prostate cancer, TRAMP TADCs are highly immunosuppressive, tolerogenic, and induce suppressive activity in tumor-specific $\mathrm{T}$ cells.

Depletion of TADCs enhances CTL survival and function. Based on our findings that TADCs tolerized $\mathrm{T}$ cells and promoted suppressor cell generation in vitro, we next sought to determine whether depletion of TADCs in vivo enhanced $T$ cell effector functions. TADC depletion was accomplished by injecting an anti-CD317 antibody, which has been previously reported to deplete pDCs (32). Prostate DCs $\left(\mathrm{CD} 11 \mathrm{c}^{+} / \mathrm{CD} 317^{+}\right.$cells) were depleted in TRAMP and WT mice for up to 18 days after i.p. injection of the anti-CD317 antibody (Supplemental Figure 5, A and B). Only B220 $0^{+}$DCs were depleted from the prostates of TRAMP mice. Significantly more TcR-I T cells were observed to infiltrate TRAMP prostates after TADC depletion (Supplemental Figure 5C), suggesting that in the absence of TADCs, TcR-I T cells underwent greater expansion and/or had increased survival. i.p. injection of anti-CD317 did deplete pDCs (CD11 $\mathrm{c}^{+} /$ $\mathrm{CD} 17^{+}$cells) but not cDCs $\left(\mathrm{CD} 11 \mathrm{c}^{+} / \mathrm{CD} 317^{-}\right.$cells) in the spleen (Supplemental Figure 5D). We next sought to determine whether in vivo depletion of TADCs enhanced TcR-I effector function. Six days after transfer, $T$ cells isolated from TADC-depleted TRAMP mice secreted significantly more IFN- $\gamma$ and granzyme B (Figure 4A) compared with that of undepleted mice. Furthermore, depletion of TADCs in TRAMP mice led to a significant reduction in suppressive activity by TcR-I cells 6 days after transfer (Figure 4B). By 12 days after $T$ cell transfer, granzyme $B$ secretion was diminished but IFN- $\gamma$ secretion was sustained at elevated levels (Figure 4C) and suppressive activity was reduced (Figure 4D). Consistent with retained antitumor activities and diminished suppressive activity, the total urogenital tract (UGT) and prostate weights, which serve as an indicator of tumor burden in the TRAMP model, were significantly lower in TADC-depleted TRAMP mice compared with those in control Ig-treated mice (Figure 4E). Taken together, these data demonstrate that TADCs in prostate tumors were involved in inducing $T$ cell tolerance and suppressive activity and are thus critical targets for ablating suppression of antitumor immunity.
Blocking TADC-derived suppressive mediators delays tolerance induction. Selective amino acid catabolism is a previously described mechanism of immune dysfunction in cancer (33-35). Our gene expression analysis demonstrated that TRAMP TADCs expressed elevated levels of Ido1 and Arg1 compared with those of WT DCs. Therefore, the role of these catabolic enzymes was tested by supplementing the drinking water of mice with 1-methyl-D-tryptophan (1MDT) or (S)-(2-boronoethyl)-L-cysteine (BEC), inhibitors of IDO and ARG1 and ARG2, respectively. When TRAMP mice were treated with these inhibitors prior to TcR-I T cell transfer, each significantly increased the ability of prostate-infiltrating TcR-I T cells to secrete IFN- $\gamma$ and granzyme B (Figure 5A). Interestingly, a gradual decay in TcR-I T cell responsiveness was noted over time, resulting in loss of antigen responsiveness after 12 days of treatment, suggesting that multiple mechanisms are responsible for induction of tolerance in the TRAMP model. Similarly, blocking IDO in vitro enhanced TcR-I proliferation in response to TADCs and reduced $T$ cell suppressive activity but did not prevent them from becoming tolerized after longer coculture (Supplemental Figure $6, \mathrm{~A}-\mathrm{C}$ ). Due to in vitro toxicity of BEC, we tested the ability of another inhibitor of ARG1 and ARG2, Nor-NOHA, to block in vitro tolerization. However, blocking ARG1 and ARG2 with NorNOHA did not enhance TcR-I responsiveness (data not shown), suggesting that our studies blocking ARG1 and ARG2 in vivo may have enhanced $T$ cell effector functions through targets other than TADC, presumably macrophages.

To test whether programmed death 1 (PD-1) ligation contributed to tolerization of TcR-I T cells, an anti-PD-1 antibody was injected after T cell transfer and TcR-I T cells were isolated on days 6 and 12 after transfer for assessment of CTL function. Blocking PD-1 in vivo delayed $T$ cell tolerance induction when tested 6 days after $\mathrm{T}$ cell transfer; $\mathrm{T}$ cells isolated from the anti-PD-1-treated group produced significantly more IFN- $\gamma$ and granzyme B compared with that of isotype control antibody-treated mice (Figure $5 \mathrm{~B}$ ). By day 12 after transfer, $T$ cells were again observed to be hyporesponsive (data not shown). In addition, blocking PD-1 in vitro increased TADC-stimulated TcR-I proliferation and reduced sup- 
A

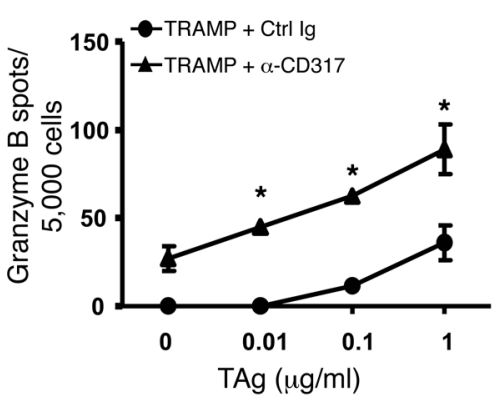

C

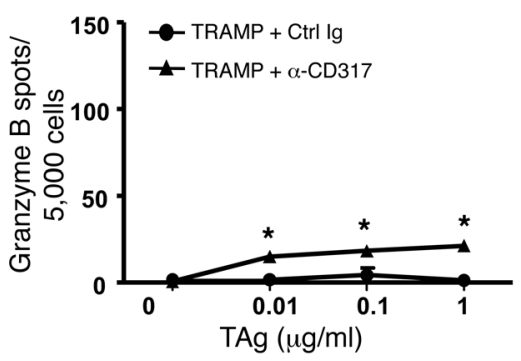

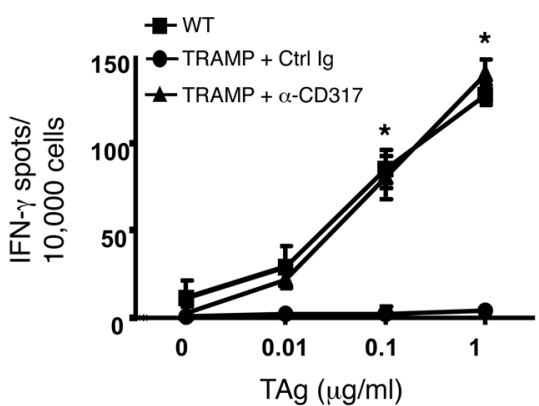

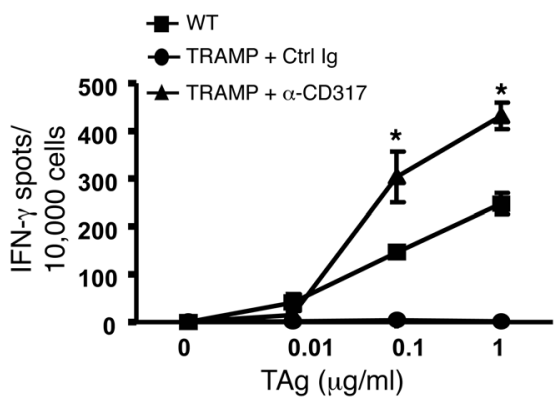

B
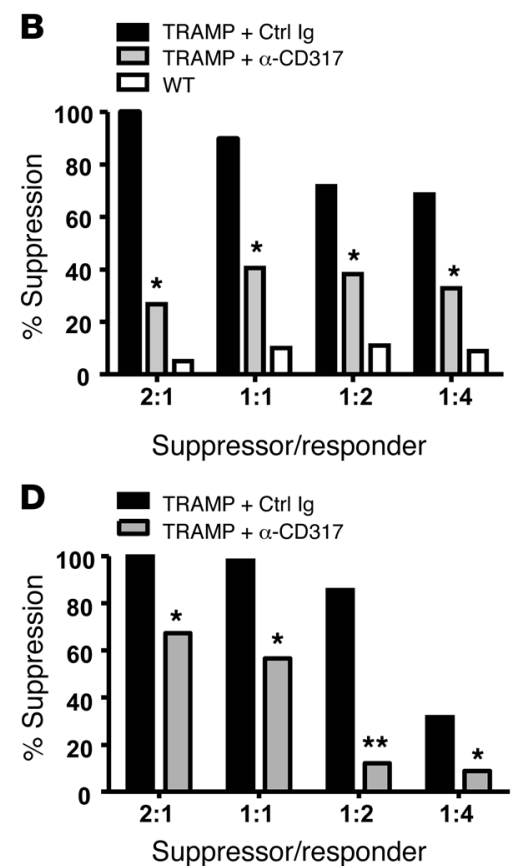

$\mathbf{E}$

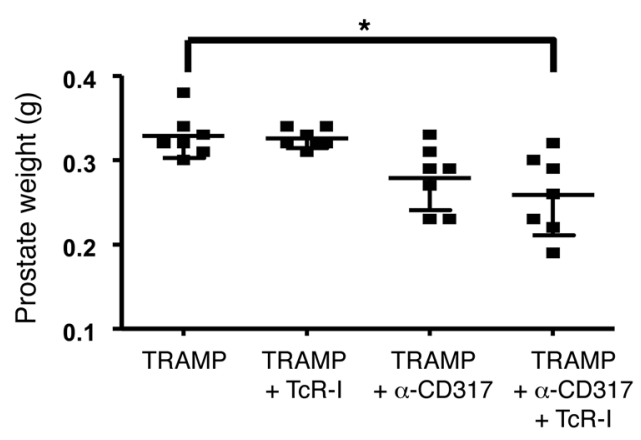

Figure 4

Depleting TADCs in TRAMP mice prevents TCR-I tolerance induction, delays suppressor cell generation, and reduces tumor burden. TADCs were depleted via i.p. injection of anti-CD317 antibody. TcR-I T cells were then transferred into TRAMP mice. T cells were reisolated from tumors (A and $\mathbf{B}) 6$ or (C and $\mathbf{D}) 12$ days after transfer to assess (A and $\mathbf{C})$ CTL effector function or (B and $\mathbf{D})$ suppressive activity. Data are representative of 3 independent trials, with 3-5 mice per group (mean \pm SD). ${ }^{*} P<0.001,{ }^{* *} P<0.0001$. Ctrl, control. (E) Weights of total UGT and dissected prostates were obtained at day 12 (mean \pm SEM). Symbols represent individual mice. ${ }^{*} P<0.01$ (Student's $t$ test). See also Supplemental Figure 5 .

pressive activity but did not prevent tolerance during the 4-day coculture (Supplemental Figure 6, B-D). These data suggest that blockade of PD-1/PD-L1 signaling enhanced the responsiveness and prolonged activation of $\mathrm{CD}^{+} \mathrm{T}$ cells stimulated by TADCs but was not sufficient to prevent tolerization. We also tested whether PD-1 blockade in combination with inhibiting IDO enhanced T cell effector functions and prevented tolerance induction, but no additive effect was observed (Supplemental Figure 6E).

TGF- $\beta$ is a pleiotropic cytokine known to induce immune suppression (36) and was upregulated in TRAMP TADCs (Table 1 and Supplemental Figure 3C). To assess the role of TGF- $\beta$ on the induction of $\mathrm{T}$ cell tolerance and suppressive activity in vivo, we treated mice with an anti-TGF- $\beta$ antibody prior to TcR-I cell transfer. TcR-I T cells isolated from the anti-TGF- $\beta$-treated mice secreted significantly more granzyme $B$ than $T$ cells from mice treated with a control antibody; surprisingly, IFN- $\gamma$ expression was not affected by TGF- $\beta$ blockade (Figure 5C). Furthermore, TcR-I
T cells from the anti-TGF- $\beta$-treated group displayed significantly less suppressive activity than the control antibody-treated group (Figure 5D). Similar to IDO and PD- 1 blockade, anti-TGF- $\beta$ added to in vitro cultures enhanced TADC-stimulated TcR-I cell proliferation and blocked suppressive activity but did not prevent tolerance during coculture (Supplemental Figure 6, B, C, and F). These data demonstrate that TGF- $\beta$ was involved in the development of TcR-I suppressor cells, but its exact role in inducing T cell tolerance will require further study.

Given the enhanced effector functions seen after inhibitory molecule blockade, the effect of blocking these suppressive mediators on antitumor immunity was tested. Interestingly, in combination with TcR-I cell transfer, treatment with either catabolic enzyme inhibitors (BEC or 1MDT) or with blocking antibodies directed against TGF- $\beta$ or PD- 1 led to a statistically significant decrease in total UGT weight and prostate weight (Supplemental Figure 6, G and H). These data suggest that after blockade of suppressive mediators, the TcR-I 


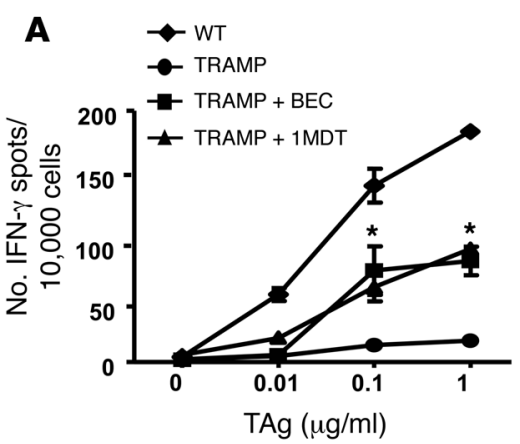

B
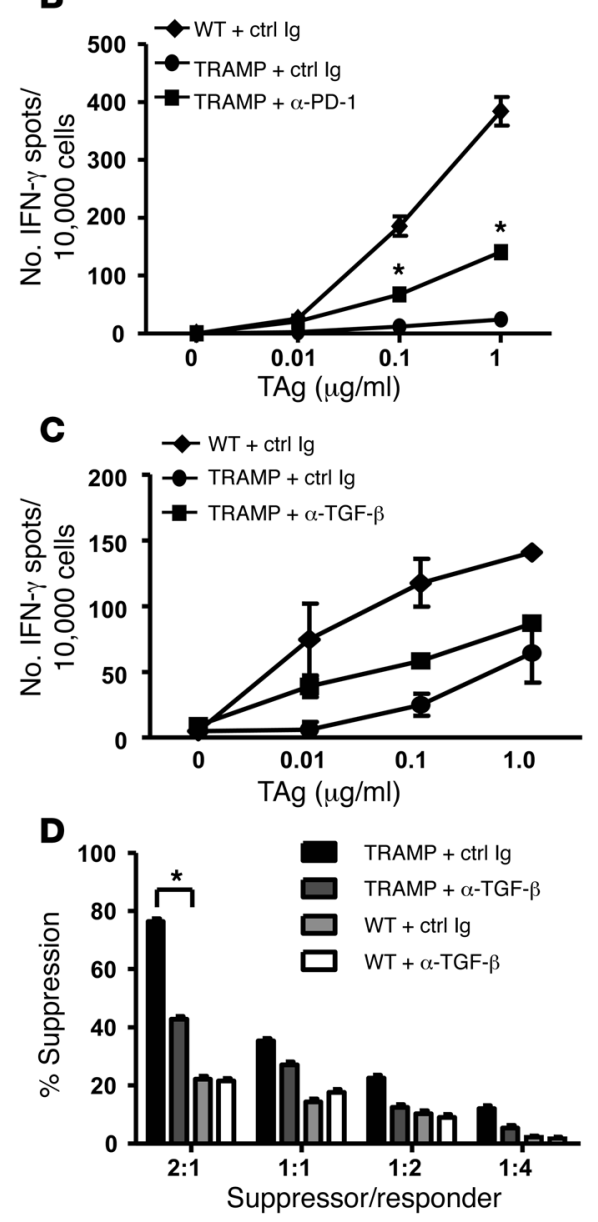

Figure 5

Blocking suppressive factors in vivo delays T cell tolerance. (A) Enzyme inhibitors 1MDT or BEC were added to the drinking water to inhibit IDO and arginase activity, respectively. (B) Mice were injected i.p. with anti-PD-1 antibody on days $0,1,3$, and 5 relative to $T$ cell transfer. (C) Mice were injected i.p. with anti-TGF- $\beta$ on days $-1,0$, and 3 relative to $T$ cell transfer. $T$ cells were harvested on day 6 after transfer to assess IFN- $\gamma$ (not significant) and granzyme B secretion. Data are representative of 3 independent trials (mean $\pm \mathrm{SD}$ ). ${ }^{*} P<0.05,{ }^{* *} P<0.001$ (Student's $t$ test). (D) After TGF- $\beta$ blockade in vivo, TcR-I suppressor activity was measured. 3-5 mice per group were used (mean $\pm \mathrm{SD})$. ${ }^{\star} P<0.05$. See also Supplemental Figure 6.

T cells initially infiltrate the prostate with effector functions capable of slowing tumor growth but eventually become tolerized and lose this ability, resulting in restoration of tumor growth.

FOXO3 signaling pathway programs TADCs to tolerize $T$ cells. We recently reported that a proinflammatory environment augmented the stimulatory capacity of TADCs (22). These findings suggest that the plasticity of TADCs may be a programmable function regulated by transcriptional control. FOXO3 is a transcriptional regulator known to control cell cycle and activation. It was recently demonstrated that Foxo3 deficiency resulted in hyperstimulatory DCs (25). Given our observation that TRAMP TADCs overexpress of Foxo 3 relative to WT DCs or DCs isolated from younger TRAMP prostates prior to tumor development (Table 1 and Supplemental Figure 7, A-D), we hypothesized that FOXO3 may control TADC tolerogenicity. Using siRNA to silence expression, FOXO3 protein levels were slightly reduced at 24 hours but were reduced to the low levels detected in the WT prostate DCs 48 hours after siRNA transfection (Supplemental Figure 7E). TADCs treated with Foxo3 siRNAs were used to stimulate naive TcR-I T cells. In contrast to TADCs treated with control siRNAs, which are poorly stimulatory, the Foxo3 siRNA-treated TADCs were capable of inducing both strong proliferative (Supplemental Figure 7F) and cytokine (Supplemental Figure 7G) responses by TcR-I T cells. Moreover, Foxo3-silenced TADCs did not tolerize or induce suppressive activity in TcR-I cells (Figure 6, A and $\mathrm{B}$ ). These findings demonstrate that silencing expression of Foxo 3 restored their capacity to stimulate more potent antitumor responses and reduced their tolerogenicity.

To determine whether Foxo3 regulation of DC tolerogenicity is unique to prostate tumors, we assessed $\mathrm{FOXO} 3$ expression and function in pDCs isolated from other tumor models. $\mathrm{CD} 317^{+} / \mathrm{CD} 11 \mathrm{c}^{+}$cells isolated from B16 melanoma tumors had elevated levels of FOXO3 comparable to those of TRAMP TADCs (Supplemental Figure $8 \mathrm{~A}$ ) and induced $\mathrm{T}$ cell tolerance in TcR-Mel T cells (Supplemental Figure 8B). Silencing Foxo3 prevented induction of $\mathrm{T}$ cell tolerance (Supplemental Figure 8B). Similar results were obtained from TADCs isolated from orthotopic renal (RENCA) tumors (data not shown).

To understand how silencing Foxo3 resulted in greater immunostimulatory function of TADCs, changes in TADC phenotype were examined. Silencing Foxo3 enhanced CD80 expression but did not impact CD86 levels (Figure 6C). Further analysis revealed that reducing Foxo3 levels markedly decreased expression of Ido1 and Arg1 and even further increased expression of Il6 (Figure 6D), a pleiotropic cytokine associated with $\mathrm{T}$ cell survival and inflammation (37). Additionally, production of TGF- $\beta$ was completely abrogated upon silencing of 
A

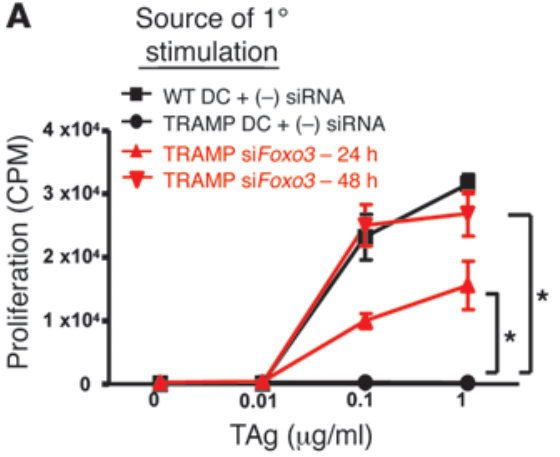

B

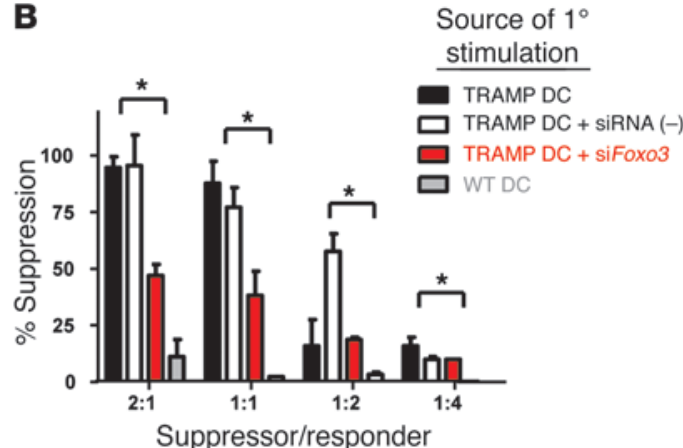

C

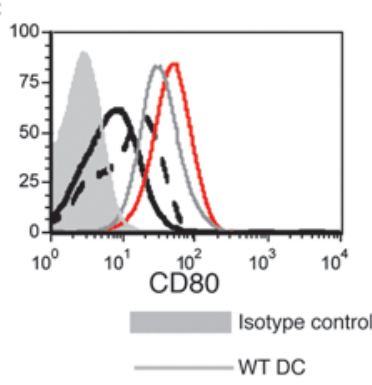

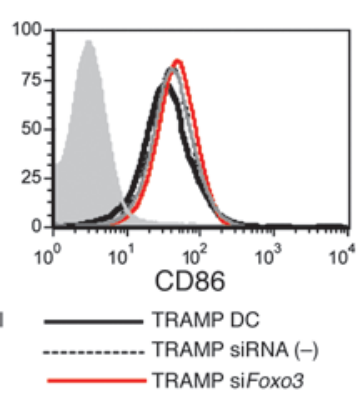

D

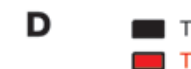

TRAMP + SIRNA (-)

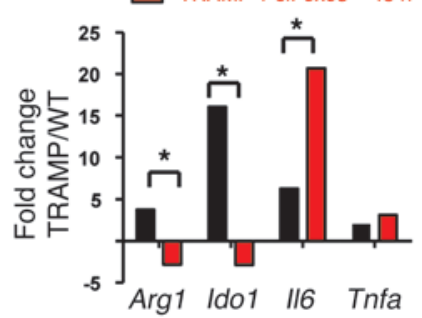

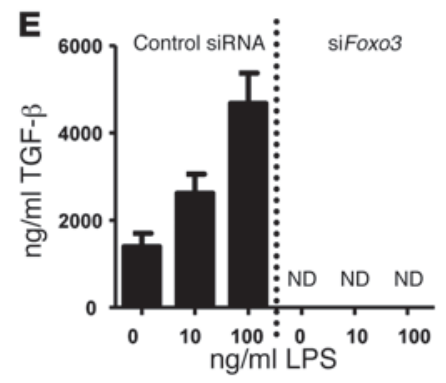
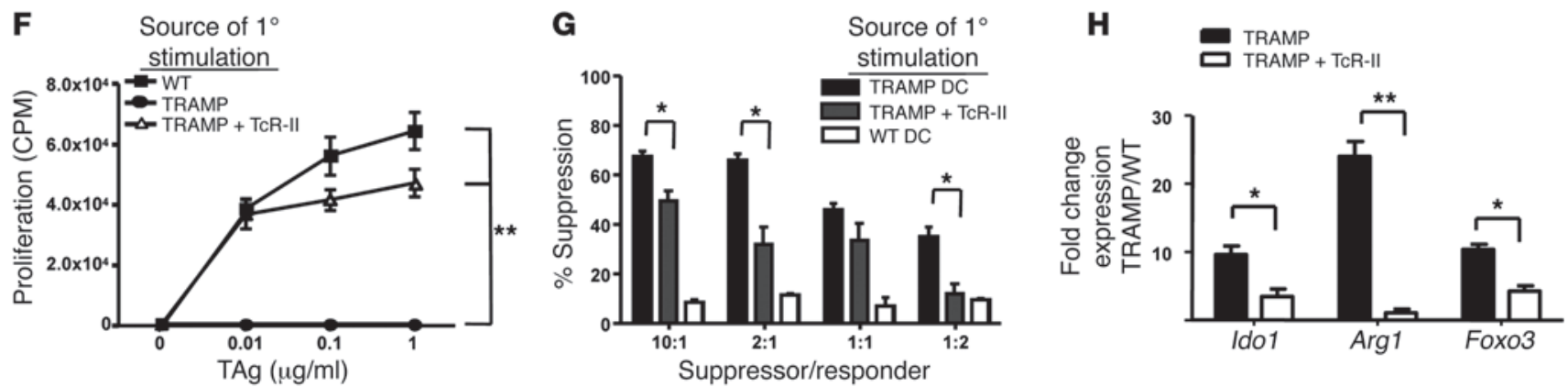

\section{Figure 6}

Silencing Foxo3 in TRAMP TADCs prevents DC-induced T cell tolerance and suppressive activity. (A) Tolerization of T cells was tested 4 days after stimulation with TADCs, as measured by proliferation and (B) suppressor activity. Silencing Foxo3 expression in TRAMP DCs induced an increase in (C) CD80 and (D) //6 expression, with a simultaneous decrease in /do1 and Arg1 expression and (E) TGF- $\beta$ production (ND, not detectable). Naive TcR-I T cells were cultured in vitro with prostatic DCs from WT or TRAMP mice transferred with TcR-II cells and tested for (F) proliferative response and (G) suppressive activity. (H) TADCs from these mice exhibited lower levels of Ido1, Arg1, and Foxo3 gene expression. Data are representative of 3 independent experiments, using 3 mice for each group (mean $\pm \mathrm{SD}$ ). ${ }^{*} P<0.01,{ }^{* *} P<0.0001$ (Student's $t$ test). See also Supplemental Figures 7 and 8. siFOXO3, Foxo3-specific siRNA; siRNA(-), scrambled control siRNA.

Foxo3 (Figure 6E). This drastic reduction in TGF- $\beta$ may explain the profound reduction of TADC-induced suppressive activity by $\mathrm{CD}^{+} \mathrm{T}$ cells (Figure $\left.6 \mathrm{~B}\right)$. These data demonstrate that targeting Foxo3 signaling in TADCs downregulates several aspects of DC function related to immune suppression, consistent with enhancing antitumor immunity.

Our previous studies demonstrated that provision of activated TAg-specific CD4 ${ }^{+}$(TcR-II) T cells increased TRAMP TADC stimulatory function (22). We now demonstrate that TADCs isolated from TRAMP mice receiving an adoptive transfer of TcR-II cells were unable to tolerize TcR-I cells in vitro (Figure 6F). To our surprise, naive TcR-I cells cultured with TADCs from TcR-II-transferred mice still maintained some suppressive functions, albeit to a lesser degree than TcR-I T cells cultured with TADCs from unmanipulated TRAMP mice (Figure 6G). Transfer of TcR-II T cells significantly reduced expression of Ido1, Arg1, and Foxo3 in DCs from the TRAMP prostate (Figure 6H). However, Foxo3 levels were not reduced to the level of that in WT prostate DCs, which might explain the residual ability to induce suppression by TcR-I cells. To confirm that TcR-II cells directly act on TADCs to alter their function, TADC cells were cultured with TcR-II cells and subsequently tested for tolerogenicity and gene expression. Coculture with TcR-II cells diminished the ability of TADCs to induce tolerance in $\mathrm{CD}^{+}$ T cells (Supplemental Figure 9A). Furthermore, TcR-II-stimulated DCs had a significant reduction in expression of Foxo3, Ido1, and Arg1 (Supplemental Figure 9B). Taken together, these data demonstrate that TADCs can be targeted in vivo to support enhanced $\mathrm{T}$ cell activation and effector functions, and inhibition of Foxo3 expression may significantly influence tolerogenicity of TADCs.

To assess the role of Foxo3 in the generation of tolerogenic TADCs in vivo, B16 tumors were injected subcutaneously into WT and $\mathrm{Foxo3}^{-/-}$mice. TADCs were isolated via magnetic beads cou- 
A
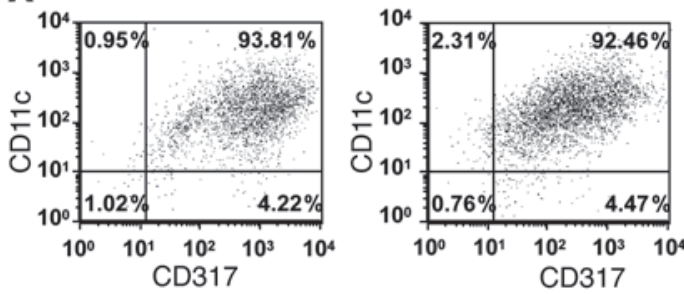

C

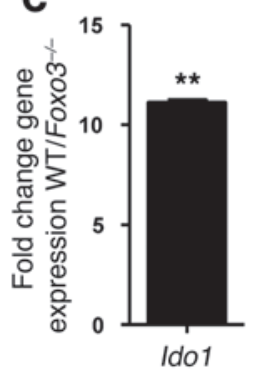

B
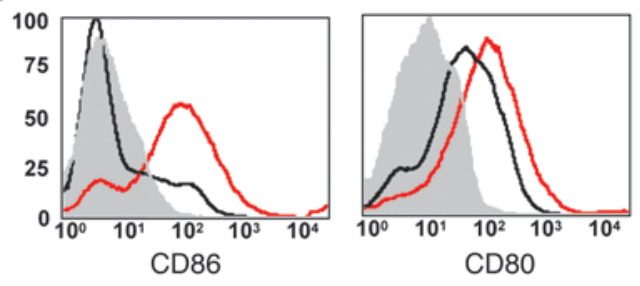

sotype control

WT B16 TADC

FoxO3 $\div$ B16 TADC

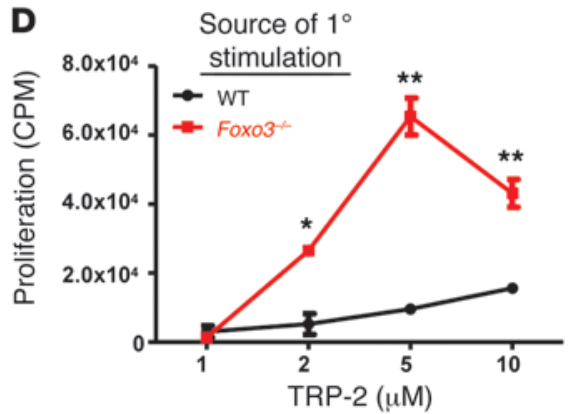

\section{Figure 7}

Foxo3--- TADCs do not induce T cell tolerance. (A) CD317+ TADCs were isolated from B16 tumors in WT or Foxo3 $3^{-/-}$mice. Numbers represent the percentages of cells present in the given quadrant. CD317+ cells isolated from B16 tumor-bearing Foxo $3^{-/-}$mice had $(B)$ elevated levels of CD80 and CD86 and (C) lower levels of Ido1 as compared with cells isolated from tumor-bearing WT mice. (D) Four days after stimulation by B16 TADCs, cell proliferation responses were tested using antigen-pulsed splenocytes. Data are representative of 3 independent experiments, using 5 mice for each group (mean $\pm \mathrm{SD}$ ). ${ }^{\star} P<0.01,{ }^{* \star} P<0.0001$ (Student's $t$ test). See also Supplemental Figure 8.

pled to anti-CD317 (Figure 7A). Phenotypically, TADCs from B16 tumors growing in $\mathrm{FoxO}^{-/-}$mice displayed elevated levels of CD80 and CD86 and lower levels of Ido1 gene expression compared with that of TADCs isolated from B16 tumors growing in WT mice (Figure $7, \mathrm{~B}$ and $\mathrm{C})$. Furthermore, TADCs isolated from the Foxo3 $3^{-1}$ mice did not tolerize TcR-Mel T cells (Figure 7D). Collectively, our in vitro data using siRNA to silence Foxo3 and our in vivo data on TADCs from Foxo $3^{-/-}$mice strongly support the hypothesis that FOXO3 is a critical regulator of DC tolerogenicity.

FOXO3 expression confers tolerogenicity to buman TADCs. The unique pattern of gene expression and function in TRAMP TADCs led us to determine whether a similar profile existed in human TADCs. We performed microarray analyses, comparing the profiles of $\mathrm{CD} 23^{+} \mathrm{pDCs}$ isolated from tumor and non-tumor specimens. Our data demonstrate a profile consistent with that observed with TRAMP TADCs. Namely, a significant upregulation of immunosuppressive genes, including FASLG, IDO1, CD274, STAT3, and FOXO3, was observed in TADCs harvested from human prostate tumor biopsies compared with that in pDCs from non-tumor prostatic tissues (Table 2). qrt-PCR confirmed that human TADCs expressed approximately 8-fold higher levels of IDO1 and 40-fold higher levels of FOXO3 mRNA compared with those of pDCs isolated from non-tumor prostate biopsies; flow cytometric analysis confirmed elevated expression of PD-L1 on human prostate TADCs (Supplemental Figure 10).

To determine whether $\mathrm{FOXO} 3$ expression is required for tolerogenicity of human TADCs, we silenced $\mathrm{FOXO} 3$ expression using siRNA. As demonstrated in Figure 8A, silencing FOXO3 (>90\% reduction in gene expression) significantly enhanced TADC stimulation of $\mathrm{T}$ cells, as indicated by an increase in proliferative response relative to priming by TADCs treated with control siRNAs. Moreover, silencing FOXO3 also abrogated the ability of human TADCs to tolerize T cells and induce suppressive activity (Figure 8, B and C). Taken together, our findings demonstrate that, similar to that in TRAMP TADCs, expression of FOXO3 is critical for the immunosuppressive activities of TADCs infiltrating human prostate cancer tissues.

\section{Discussion}

CTLs are critical for the immunotherapy-induced eradication of established tumors. Although many strategies have been attempted to use CTLs for cancer therapy (38), successes have been plagued by induction of $\mathrm{T}$ cell tolerance $(13,30,39,40)$. It may be possible to prevent tolerance by providing a potent proinflammatory stimulus, such as $\mathrm{CD}^{+} \mathrm{T}$ cell help (22), or to reverse tolerance by providing cytokines (41). Interestingly, both strategies could involve the licensing or stimulation of DCs for the production of proinflammatory cytokines (IL-12 and IL-15) and increasing costimulatory molecule expression for better $\mathrm{T}$ cell stimulation.

In the current study, we demonstrate that TADCs are critical in determining the outcome of $\mathrm{T}$ cell responses within the tumor microenvironment. We demonstrate that TADCs expressed many genes associated with immune suppression, including Ido1, Arg1, and $C d 274$ (also known as Pdl1), and produced high levels of TGF- $\beta$, which may result in induction of T cell suppressive activity (31). Blockade of each pathway was able to delay tolerization of T cells and induction of suppressive activity. To our surprise, combina- 


\section{Table 2}

Human TADCs have elevated expression of genes associated with tolerance

$\begin{array}{lc}\text { Gene } & \text { Fold change tumor/non-tumor } \\ \text { FASLG } & 5.2 \\ \text { IDO1 } & 7.3 \\ \text { CD274 } & 3.1 \\ \text { STAT3 } & 5.1 \\ \text { FOX03 } & 6.9\end{array}$

RNA was isolated and hybridized to Affymetrix Human Gene 1.0 ST arrays. Fold change values have corresponding $P$ values of less than 0.00001 (ANOVA). Data are representative of 5 independent microarrays for tumor and non-tumor biopsies.

torial blockade of the pathways did not further improve $\mathrm{T}$ cell responsiveness. However, depletion of TADCs in TRAMP mice, using antibodies directed against BST2/CD317, improved $\mathrm{T}$ cell expansion, increased $\mathrm{T}$ cell effector functions for up to 2.5 weeks (at which point TADCs begin to reinfiltrate the tumor), and led to a reduction in tumor burden, demonstrating that TADCs play an active role in induction of $\mathrm{T}$ cell tolerance. However, we cannot rule out the possibility that this antibody depletes another as-yetundefined $\mathrm{CD} 317^{+}$population of regulatory cells as well, which also controls TcR-I cell expansion. While slightly different than the previous findings of Adler and colleagues (15), our results suggest that inhibition of a single or even a combination of suppressive factors may not be sufficient to improve antitumor immunity but rather that TADCs are programmed to become efficient and potent suppressors of immune function through multiple, nonredundant mechanisms. Complete elimination or "reprogramming" may be required to prevent the profound immunosuppression associated with TADCs.

Our findings are somewhat divergent from those of Norian et al., who described a population of DCs (described as "tumorinfiltrating") that tolerize T cells in an ARG-dependent mechanism (42). Those "TIDCs" did exert strong immunosuppressive effects on $\mathrm{CD}^{+} \mathrm{T}$ cells. However, the phenotype of the TIDCs was more consistent with cDCs (B220 and CD317 expression was not tested), and expression of Foxo 3 was not assessed. Taken together with other studies identifying a tolerogenic role for pDCs in cancer $(18,19)$, these findings underscore the heterogeneity of DCs that infiltrate different tumors and the need for understanding how DCs infiltrating tumors acquire their immunosuppressive functions.

Our data further identify what we believe to be a new tolerogenic programming pathway in TADCs. Foxo3 was initially identified as a tumor suppressor gene, with antiinflammatory functions associated with inhibiting NF- $\mathrm{KB}$ signals and inducing cell cycle arrest $(26,43)$. Hedrick and colleagues recently reported that B7 engagement by CTLA-4 induces expression of Foxo3, which restricts DC stimulatory capacity (25). We now report that TADCs infiltrating multiple tumors overexpress Foxo3, and we show for the first time to our knowledge that silencing Foxo3 reduced the ability of these TADCs to induce tolerance and suppressive activity in antigen-specific T cells, rendering these TADCs more immunostimulatory. Moreover, TADCs from B16 tumors growing in Foxo $3^{-/-}$mice were not tolerogenic. Thus, Foxo3 appears to be a critical component of the programming machinery that directs
TADCs to be suppressive and may be a new and potent target for enhancing immunotherapy. Our on-going studies are identifying the downstream effectors of Foxo3 as a means of identifying additional novel targets for cancer therapy as well as endogenous signals that induce Foxo3 expression.

An inhibitory role for DCs in human cancer was previously suggested by others (44-49). We have now demonstrated that a population of TADCs, with a pDC phenotype similar to that of TADCs observed in the TRAMP prostate cancer model, is present in human prostate cancer tissues. Interestingly, these TADCs were poor stimulators of $\mathrm{T}$ cells, tolerized autologous $\mathrm{T}$ cells, and overexpressed $\mathrm{FOXO} 3$ compared with pDCs from non-tumor prostatic tissues. Of considerable interest is our observation that silencing FOXO3 in human TADCs also led to increased stimulatory capacity and reduced tolerogenicity. These findings implicate FOXO3 as an important and effective target for reversing TADCinduced immunosuppression. Thus, providing signals that lead to reduced FOXO3 expression will reduce immune suppression and enhance immunity to tumor antigens.

We believe that these observations not only confirm the relevance of our findings in experimental tumor models but also support the rationale that TADCs are a suitable target for enhancing immunotherapy for cancer. For example, immunotherapies that alter the tumor microenvironment may also be a viable approach to modifying TADCs. Consistent with this idea, we recently reported that activated $\mathrm{CD}^{+} \mathrm{T}$ cells can infiltrate a tumor and activate DCs in situ (22). We now demonstrate that provision of CD4 help also resulted in decreased Foxo3 expression, increased stimulatory capacity, and loss of tolerogenicity of TRAMP TADCs. While provision of CD4 help to boost antitumor immunity is not novel, our findings have critical implications for focusing immune-based therapy on the tumor microenvironment. By providing activated $\mathrm{CD}^{+} \mathrm{T}$ cells to the tumor, either by chemokine-directed trafficking (50) or by conferring expression of chimeric receptors specific for tumor antigens (51), long-term prevention of TADC tolerogenicity may be achieved.

Strategies that block inhibitory pathways in TADCs, such as those initiated by FOXO3, may enhance antitumor immunity and prevent the profound immunosuppression observed in the microenvironment of many different types of tumors. FOXO3 signaling may represent a common mediator of immune suppression and may be responsible for the generation of IDO, arginase, TGF- $\beta$, and other as-yet-unidentified suppressive factors produced by TADCs. Consequently, these approaches will globally alter DC stimulatory capacity and may be more effective than therapies that target a particular pathway (e.g., catabolic enzymes or individual inhibitory receptors).

\section{Methods}

Experimental mice. TRAMP (52) and B6C3F1 nontransgenic control mice served as recipients for $\mathrm{T}$ cell transfer. The SV40 TAg-specific CD8 ${ }^{+} \mathrm{TcR}-\mathrm{I}$ and CD4+ TcR-II transgenic mouse strains were bred onto a Rag ${ }^{-1-}$ background as previously described (22). The TcR transgenic mouse strains $37 \mathrm{~B} 7$ and TcR-Mel bear a TcR transgene that recognizes an $\mathrm{H}-2 \mathrm{~K}^{\mathrm{b}}$-restrict-

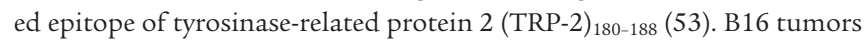
were injected into $\mathrm{Foxo3}^{-/-}$mice (provided by Stephen Hedrick, UCSD, San Diego, California, USA) (25) or C57BL/6 mice. Mice were housed under specific pathogen-free conditions and were treated in accordance with National Institutes of Health guidelines, under protocols approved by the Animal Care and Use Committee of the NCI-Frederick facility. 
A

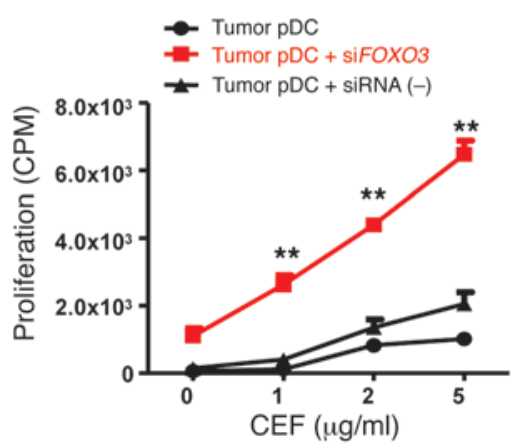

B

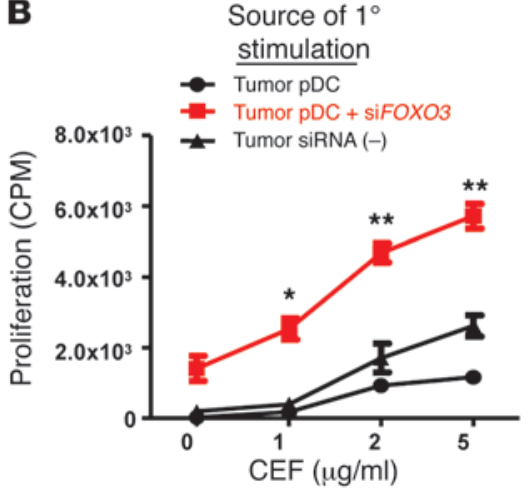

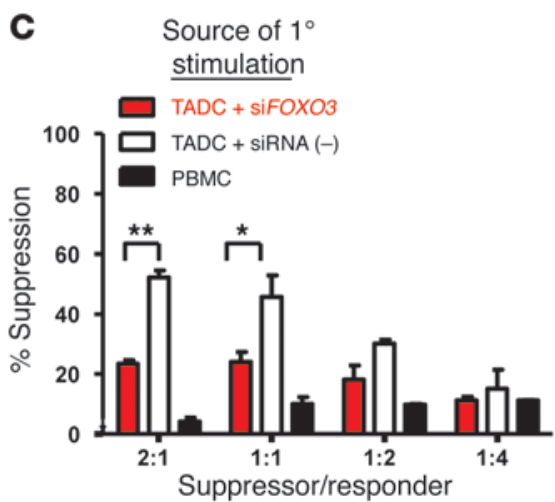

Figure 8

Silencing FOXO3 in human TADC enhances stimulation. siRNA-treated TADCs isolated from human prostate tumor tissues were used to (A) stimulate, (B) tolerize, or (C) induce suppressive activity in autologous PBMCs in vitro using the CEF viral peptide pool. Proliferation of (A) primary or (B) secondary responses was assessed using thymidine incorporation. Data are representative of (A) 3 different patient samples and (B and C) 2 patient samples (mean \pm SD). ${ }^{\star} P<0.01,{ }^{\star \star} P<0.001$ (Student's $t$-test). See also Supplemental Figure 10.

Human tissues. Human tissue specimens were obtained at the time of surgical resection. Tissues were examined by the University of Maryland Surgical Pathology Department for identification of tumor and non-tumor tissues. The use of these tissues was determined to be exempt from the federal regulations for the protection of human Subjects by the NCI Office of Human Subjects Research. No specific patient information was received. TADCs were analyzed from 16 patient samples. Biopsies were weighed, fixed for immunohistochemistry, or digested in collagenase/DNAse for 30 minutes. DCs were isolated via magnetic beads conjugated to anti-PTK7 or anti-CD304 (BDCA-4) for pDCs according to manufacture's instructions (Miltenyi Biotech). Human PBMCs were stimulated with $2 \mu \mathrm{g} / \mathrm{ml}$ $\mathrm{CD}^{+}$peptides for CEF (CEF peptide pool, Mabtech). The peptides are not all restricted by HLA A2 and react with more than $90 \%$ of people of mixed European descent.

Peptides. SV40 TAg, TAg ${ }_{560-568}$ (SEFLLEKRI), and TRP-2 $180-188$ (SVYDFFVWL) peptides were purchased from New England Peptide.

Adoptive transfer of transgenic lymphocytes. LN cells from TcR-I mice were prepared in a single-cell suspension. Cell numbers were adjusted to $3 \times 10^{6}$ TcR-I cells to be transferred i.v. into recipient mice. In some experiments, inhibitors to IDO (1MDT) and arginase (BEC) were added to the drinking water every 3 days $(5 \mu \mathrm{g} / \mathrm{ml})$.

Blocking and depletion antibodies. A blocking anti-PD-1 antibody (clone RMP1-14) (54) was injected i.p. on days 0, 1, and 3, with respect to adoptive transfer of TcR-I T cells $(250 \mu \mathrm{g} /$ injection). Anti-TGF- $\beta$ antibody was injected i.p. on days $-1,0,1$, and 2, with respect to TcR-I transfer (500 $\mu \mathrm{g} /$ injection). Anti-CD317 antibody (5 $\mu \mathrm{g}$ ) (provided by Marco Colonna, Washington University, St. Louis, Missouri, USA) was injected i.p. on days -1 and 0 , with respect to TcR-I transfer (32).

Cell isolations. Prostatic tissues were removed from 14- to 16-week-old TRAMP mice in groups of 3-6 mice or as indicated. Tissues were digested in a solution of Collagenase and DNAse. DCs were isolated from single-cell suspensions of the prostate using the Miltenyi MACS cell separation system and the Pan-DC magnetic beads, which consist of anti-CD11c and antimPDCA-1 (CD317) antibody. Cell separations were completed according to the manufacturer's instruction and consistently yielded purity of more than

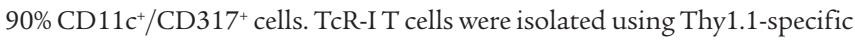
antibodies and magnetic beads as described previously (30).

Flow cytometry. Cell suspensions were blocked with Fc block, washed, and incubated with the indicated antibodies purchased from BD Pharmingen or eBioscience.
In vitro proliferation assays. Naive TcR-I T cells were used as responder cells in a proliferation assay; $2 \times 10^{4} \mathrm{~T}$ cells were stimulated with antigen and $2 \times 10^{4}$ isolated prostatic DCs. After 72 hours of culture, wells were pulsed with $1 \mu \mathrm{Ci}\left[{ }^{3} \mathrm{H}\right]$ thymidine (Amersham) for 16 hours. The cells were then harvested using a Cell Harvester (Tomtech), and radioactivity was measured in a Liquid Scintillation Counter (Trilux MicroBeta, Wallac).

Tolerance and suppression assays. Naive TcR-I or TcR-Mel T cells were cocultured with DCs for 72 hours. T cells were then reisolated via negative selection with magnetic beads. To assess tolerance, $\mathrm{T}$ cells were subjected to secondary stimulation with normal splenocytes and antigens. After 48 hours, wells were pulsed with $1 \mu \mathrm{Ci}\left[{ }^{3} \mathrm{H}\right]$ thymidine (Amersham) for 12 hours. To assess suppressive activity, graded numbers of TcR-I T cells were added to $1 \times 10^{4}$ responder $37 \mathrm{~B} 7 \mathrm{TRP}-2$-specific T cells (53), $1 \times 10^{5}$ splenocytes, and $5 \mu \mathrm{M}$ TRP- 2 and incubated for 72 hours at $37^{\circ} \mathrm{C}$. One $\mu \mathrm{Ci}$ of $\left[{ }^{3} \mathrm{H}\right]$ thymidine per well was added for an additional 16 hours and harvested as described above. Previous studies have demonstrated that in this assay tolerized T cells do not proliferate, and, therefore, ${ }^{3} \mathrm{H}$-thymidine incorporation is an indication of 37B7 cell proliferation (31).

For human assays, autologous PBMCs were cultured for 72 hours with TADCs isolated from human prostate tumor tissue or irradiated PBMCs. Lymphocytes were then reisolated by negative selection using magnetic beads. To assess tolerance, cultured lymphocytes were restimulated with irradiated autologous PBMCs and CEF antigen for 48 hours. To assess suppressive activity, cultured lymphocytes were used to suppress autologous PBMC stimulation at graded suppressor/ responder ratios for 48 hours. Wells were pulsed with $1 \mu \mathrm{Ci}\left[{ }^{3} \mathrm{H}\right]$ thymidine (Amersham) for 12 hours.

ELISPOT assays. Multiscreen plates (Millipore) were coated with $100 \mu \mathrm{l}$ of capture antibody (R\&D Systems) overnight at $4^{\circ} \mathrm{C}$. IFN- $\gamma\left(1 \times 10^{4}\right)$ or granzyme $\mathrm{B}\left(5 \times 10^{3}\right)$ purified Thy $1.1^{+}$TcR-I T cells were added to increasing concentrations of $\mathrm{TAg}_{560-568}$. After incubation, plates were washed and processed as previously described (22).

Microarray and real time PCR. RNA was isolated by RNAeasy Spin Columns (Qiagen), per manufactures instructions, from DCs purified from 5 TRAMP and 5 WT mouse prostate tissues. RNA quality was determined by NanoDrop Spectrophotometer. High quality RNA was sent to the Laboratory of Molecular Technologies (SAIC) for hybridization to Mouse 1.0 ST Gene Array or Human Affymetrix Human Gene 1.0 ST array. Gene expression and pathway analysis was performed with Partek and Gene Portal software. Fold changes greater than 2.1 and 
$P<0.0001$ were considered significant and verified by real-time PCR. Microarray data was submitted to NCBI's GEO database (http://www. ncbi.nlm.nih.gov/geo/) with accession numbers of GSE26719 (mouse) and GSE26747 (human).

Primers for RT-PCR were purchased (SA Biosciences and ABI) and used per manufactures instructions in combination with Sybr Green (SA Biosciences). Samples were run on a Bio-Rad iCycler RT-PCR machine. For each pair-wise set of samples (WT vs. TRAMP or tumor vs. non-tumor), fold change was calculated using the relative expression software tool (REST software, Corbett Research) (55).

Gene silencing. DCs were isolated from TRAMP or WT prostates and cultured with 4 mixed siRNAs to Foxo3, Gapdh, or scrambled negative control siRNAs (purchased from SA Biosciences and ABI) for 24, 48, and 72 hours. DCs were then stained for flow cytometric analysis for cell viability and activation markers (CD80, CD86, CD11c), lysed for protein for Western blot (FOXO3, $\beta$-ACTIN), lysed for RNA for qrt-PCR, or added to T cell stimulation assays. Viability of DCs after siRNA treatment was equivalent, irrespective of the siRNA used.

Statistics. Statistical analyses for differences between group means were performed by unpaired (2-tailed) Student's $t$ test or ANOVA. Data are presented as mean \pm SEM or mean \pm SD as indicated. $P<0.05$ was considered statistically significant.

\section{Acknowledgments}

The authors would like to thank Joost Oppenheim, Giorgio Trinchieri, Jose Conejo-Garcia, Jay Berzofsky, Bill Murphy, and Nicholas Restifo for their helpful discussions and critical review of this manuscript and Scott Hudson for his assistance in uploading microarray data to GEO. This work was supported by the Intramural Research Program of the NCI under award Z01 BC 010954. Additional support was provided by the Center for Cancer Research, Office of Science and Technology Partnerships, and the Department of Defense Prostate Cancer Research Program.

Received for publication July 12, 2010, and accepted in revised form January 26, 2011.

Address correspondence to: Arthur A. Hurwitz, Cancer and Inflammation Program, 211 Building 567, NCI-Frederick, Frederick, Maryland 21702, USA. Phone: 301.846.5443; Fax: 301.846.7350; E-mail: hurwitza@mail.nih.gov.

Elena Riboldi's present address is: Dipartimento di Scienze Chimiche, Alimentari, Farmaceutiche e Farmacologiche (DISCAFF), University of Piemonte Orientale A. Avogadro, Novara, Italy.
1. Chaput N, Conforti R, Viaud S, Spatz A, Zitvogel L. The Janus face of dendritic cells in cancer. Oncogene. 2008;27(45):5920-5931.

2. Kusmartsev S, Gabrilovich DI. Role of immature myeloid cells in mechanisms of immune evasion in cancer. Cancer Immunol Immunother. 2006; 55(3):237-245.

3. Watkins SK, Egilmez NK, Suttles J, Stout RD. IL-12 rapidly alters the functional profile of tumor-associated and tumor-infiltrating macrophages in vitro and in vivo. J Immunol. 2007;178(3):1357-1362.

4. Hou DY, et al. Inhibition of indoleamine 2,3dioxygenase in dendritic cells by stereoisomers of 1-methyl-tryptophan correlates with antitumor responses. Cancer Res. 2007;67(2):792-801.

5. Mellor AL, Munn DH. IDO expression by dendritic cells: tolerance and tryptophan catabolism. Nat Rev Immunol. 2004;4(10):762-774.

6. Qiu CH, Miyake Y, Kaise H, Kitamura H, Ohara O, Tanaka M. Novel subset of CD8\{alpha\}+ dendritic cells localized in the marginal zone is responsible for tolerance to cell-associated antigens. J Immunol. 2009;182(7):4127-4136.

7. Stoitzner P, et al. Inefficient presentation of tumor-derived antigen by tumor-infiltrating dendritic cells. Cancer Immunol Immunother. 2008; 57(11):1665-1673.

8. Fallarino $\mathrm{F}$, et al. $\mathrm{T}$ cell apoptosis by kynurenines. Adv Exp Med Biol. 2003;527:183-190.

9. Muller AJ, Prendergast GC. Indoleamine 2,3-dioxygenase in immune suppression and cancer. Curr Cancer Drug Targets. 2007;7(1):31-40.

10. Norian LA, et al. Tumor-infiltrating regulatory dendritic cells inhibit CD8+ T cell function via L-arginine metabolism. Cancer Res. 2009;69(7):3086-3094.

11. Keir ME, Francisco LM, Sharpe AH. PD-1 and its ligands in T-cell immunity. Curr Opin Immunol. 2007;19(3):309-314.

12. Latchman YE, et al. PD-L1-deficient mice show that PD-L1 on T cells, antigen-presenting cells, and host tissues negatively regulates T cells. Proc Natl Acad SciUS A. 2004;101(29):10691-10696.

13. Adler AJ, et al. CD4+ T cell tolerance to parenchymal self-antigens requires presentation by bone marrow-derived antigen-presenting cells. J Exp Med. 1998;187(10):1555-1564.

14. Sotomayor EM, et al. Cross-presentation of tumor antigens by bone marrow-derived antigen-present- ing cells is the dominant mechanism in the induction of T-cell tolerance during B-cell lymphoma progression. Blood. 2001;98(4):1070-1077.

15. Hagymasi AT, et al. Steady state dendritic cells present parenchymal self-antigen and contribute to, but are not essential for, tolerization of naive and Th1 effector CD4 cells. J Immunol. 2007; 179(3):1524-1531.

16. Munn DH, et al. Expression of indoleamine 2,3dioxygenase by plasmacytoid dendritic cells in tumor-draining lymph nodes. J Clin Invest. 2004; 114(2):280-290.

17. Rabinovich GA, Gabrilovich D, Sotomayor EM. Immunosuppressive strategies that are mediated by tumor cells. Annu Rev Immunol. 2007;25:267-296.

18. Treilleux I, et al. Dendritic cell infiltration and prognosis of early stage breast cancer. Clin Cancer Res. 2004;10(22):7466-7474.

19. Wei S, et al. Plasmacytoid dendritic cells induce CD8+ regulatory $\mathrm{T}$ cells in human ovarian carcinoma. Cancer Res. 2005;65(12):5020-5026.

20. Zou W, et al. Stromal-derived factor-1 in human tumors recruits and alters the function of plasmacytoid precursor dendritic cells. Nat Med. 2001; 7(12):1339-1346.

21. Lipscomb MW, et al. Ectopic T-bet expression licenses dendritic cells for IL-12-independent priming of type $1 \mathrm{~T}$ cells in vitro. J Immunol. 2009; 183(11):7250-7258

22. Shafer-Weaver KA, et al. Immunity to murine prostatic tumors: continuous provision of T-cell help prevents CD8 T-cell tolerance and activates tumor-infiltrating dendritic cells. Cancer Res. 2009; 69(15):6256-6264.

23. Cheng F, et al. A critical role for Stat 3 signaling in immune tolerance. Immunity. 2003;19(3):425-436.

24. Yu H, Pardoll D, Jove R. STATs in cancer inflammation and immunity: a leading role for STAT3. Nat Rev Cancer. 2009;9(11):798-809.

25. Dejean AS, et al. Transcription factor Foxo3 controls the magnitude of $\mathrm{T}$ cell immune responses by modulating the function of dendritic cells. Nat Immunol. 2009;10(5):504-513.

26. Essers MA, de Vries-Smits LM, Barker N, Polderman PE, Burgering BM, Korswagen HC. Functional interaction between beta-catenin and FOXO in oxidative stress signaling. Science. 2005;308(5725):1181-1184.
27. Perez L, Shurin MR, Collins B, Kogan D, Tourkova IL, Shurin GV. Comparative analysis of CD1a, S100, CD83, and CD11c human dendritic cells in normal, premalignant, and malignant tissues. Histol Histopathol. 2005;20(4):1165-1172.

28. Troy AJ, Summers KL, Davidson PJ, Atkinson CH, Hart DN. Minimal recruitment and activation of dendritic cells within renal cell carcinoma. Clin Cancer Res. 1998;4(3):585-593.

29. Colonna M, Trinchieri G, Liu YJ. Plasmacytoid dendritic cells in immunity. Nat Immunol. 2004; 5(12):1219-1226.

30. Anderson MJ, Shafer-Weaver K, Greenberg NM, Hurwitz AA. Tolerization of tumor-specific T cells despite efficient initial priming in a primary murine model of prostate cancer. J Immunol. 2007; 178(3):1268-1276.

31. Shafer-Weaver KA, Anderson MJ, Stagliano K, Malyguine A, Greenberg NM, Hurwitz AA. Cutting Edge: Tumor-specific CD8+ T cells infiltrating prostatic tumors are induced to become suppressor cells. J Immunol. 2009;183(8):4848-4852.

32. Blasius AL, Giurisato E, Cella M, Schreiber RD, Shaw AS, Colonna M. Bone marrow stromal cell antigen 2 is a specific marker of type I IFN-producing cells in the naive mouse, but a promiscuous cell surface antigen following IFN stimulation. J Immunol. 2006;177(5):3260-3265.

33. Bronte $\mathrm{V}$, et al. Boosting antitumor responses of $\mathrm{T}$ lymphocytes infiltrating human prostate cancers. J Exp Med. 2005;201(8):1257-1268.

34. Sharma MD, et al. Plasmacytoid dendritic cells from mouse tumor-draining lymph nodes directly activate mature Tregs via indoleamine 2,3-dioxygenase. J Clin Invest. 2007;117(9):2570-2582.

35. Srivastava MK, Sinha P, Clements VK, Rodriguez P, Ostrand-Rosenberg S. Myeloid-derived suppressor cells inhibit T-cell activation by depleting cystine and cysteine. Cancer Res. 2010;70(1):68-77.

36. Li MO, Wan YY, Sanjabi S, Robertson AK, Flavell RA. Transforming growth factor-beta regulation of immune responses. Annu Rev Immunol. 2006;24:99-146.

37. Marrack P, et al. T-cell survival. Immunol Rev. 1998;165:279-285.

38. June $\mathrm{CH}$. Adoptive $\mathrm{T}$ cell therapy for cancer in the clinic. J Clin Invest. 2007;117(6):1466-1476.

39. Zhang Y, Huang S, Gong D, Qin Y, Shen Q. Pro- 
grammed death-1 upregulation is correlated with dysfunction of tumor-infiltrating CD8(+) T lymphocytes in human non-small cell lung cancer. Cell Mol Immunol. 2010;7(5):389-395.

40. Zhou Q, et al. Program death-1 (PD-1) signaling and regulatory $\mathrm{T}$ cells (Tregs) collaborate to resist the function of adoptively transferred cytotoxic $\mathrm{T}$ lymphocytes (CTLs) in advanced acute myeloid leukemia (AML). Blood. 2010;116(14):2484-2493.

41. Teague RM, et al. Interleukin-15 rescues tolerant CD8 + T cells for use in adoptive immunotherapy of established tumors. Nat Med. 2006;12(3):335-341.

42. Norian LA, et al. Tumor-infiltrating regulatory dendritic cells inhibit CD8+ T cell function via L-arginine metabolism. Cancer Res. 2009; 69(7):3086-3094.

43. Shang YC, Chong ZZ, Hou J, Maiese K. The forkhead transcription factor FOXO3a controls microglial inflammatory activation and eventual apoptotic injury through caspase 3 . Curr Neurovasc Res. 2009;6(1):20-31.

44. Aalamian $\mathrm{M}$, et al. Human prostate cancer regulates generation and maturation of monocyte-derived dendritic cells. Prostate. 2001;46(1):68-75.

45. Almand B, et al. Clinical significance of defective dendritic cell differentiation in cancer. Clin Cancer Res. 2000;6(5):1755-1766.

46. Banerjee DK, Dhodapkar MV, Matayeva E, Steinman RM, Dhodapkar KM. Expansion of FOXP3high regulatory $\mathrm{T}$ cells by human dendritic cells (DCs) in vitro and after injection of cytokine-matured DCs in myeloma patients. Blood. 2006;108(8):2655-2661.

47. Enk AH, Jonuleit H, Saloga J, Knop J. Dendritic cells as mediators of tumor-induced tolerance in metastatic melanoma. Int J Cancer. 1997;73(3):309-316.

48. Esche C, et al. Tumor's other immune targets: dendritic cells. J Leukoc Biol. 1999;66(2):336-344.

49. Gabrilovich DI, Corak J, Ciernik IF, Kavanaugh D, Carbone DP. Decreased antigen presentation by dendritic cells in patients with breast cancer. Clin Cancer Res. 1997;3(3):483-490.

50. Gough M, et al. Gene therapy to manipulate effector $\mathrm{T}$ cell trafficking to tumors for immunotherapy.
J Immunol. 2005;174(9):5766-5773.

51. Pinthus JH, et al. Immuno-gene therapy of established prostate tumors using chimeric receptorredirected human lymphocytes. Cancer Res. 2003; 63(10):2470-2476.

52. Greenberg NM, et al. Prostate cancer in a transgenic mouse. Proc Natl Acad Sci U S A. 1995; 92(8):3439-3443

53. Singh V, Ji Q, Feigenbaum L, Leighty RM, Hurwitz AA. Melanoma progression despite infiltration by in vivo-primed TRP-2-specific T cells. J Immunother. 2009;32(2):129-139.

54. Yamazaki T, Akiba H, Koyanagi A, Azuma M, Yagita H, Okumura K. Blockade of B7-H1 on macrophages suppresses $\mathrm{CD} 4+\mathrm{T}$ cell proliferation by augmenting IFN-gamma-induced nitric oxide production. J Immunol. 2005;175(3):1586-1592.

55. Pfaffl MW, Horgan GW, Dempfle L. Relative expression software tool (REST) for group-wise comparison and statistical analysis of relative expression results in real-time PCR. Nucleic Acids Res. 2002;30(9):e36. 